\title{
Process optimization for yogurt preparation incorporated with encapsulated Caesalpinia bonducella Flem. seed extract
}

\author{
Sadhna Mishra, Arvind ${ }^{\star}$ Dinesh Chandra Rai and Shikha Pandhi \\ Department of Dairy Science and Food Technology, Institute of Agricultural Sciences, Banaras Hindu University, Varanasi-221005, Uttar Pradesh, \\ India
}

\section{Article Info}

Article history

Received 23 April 2020

Revised 13 June 2020

Accepted 15 June 2020

Published online 30 June 2020

\section{Keywords}

Yogurt

Caesalpinia bonducella Flem.

Antioxidant

Encapsulation

\begin{abstract}
The present study was conducted to optimize the process for the preparation of yogurt incorporated with Caesalpinia bonducella Flem. seeds extract. The process of ultrasonication was used for extraction followed by encapsulation using a spray drier. Extracts were encapsulated (to enhance their endurance and hydrophilicity) by spray-drying (at optimized $160^{\circ} \mathrm{C}$ inlet and $80^{\circ} \mathrm{C}$ outlet temperatures), using an optimized 1:2 ratio of sodium alginate cross linked with inulin as wall material. Both the aqueous and encapsulated extract was used for incorporation into yogurt and optimized based on antioxidant activity, total phenolic contents, sensory and texture analysis. The sample of yogurt with $3 \%$ fat and $2 \%$ aqueous extract had highest values of $\%$ DPPH inhibition (88.82\%), ABTS inhibition activity (93.23\%), SOSA (85.54\%) and TPC (25.77 $\mathrm{mg} \mathrm{GA}$ eq./g) while the yogurt sample with $3 \%$ fat and $2 \%$ encapsulated powder was found best in terms of sensory profile and texture among other variants. The total polyphenolic contents increased proportionally with increasing levels of encapsulated as well as non encapsulated extract.
\end{abstract}

\section{Introduction}

The fermented food products are the most popular foodstuffs in the world as they have been coupled with many health benefits and high nutritional value due to the presence of beneficial live microorganisms, proteins, and vitamins A, B, C, D, and E (Ares et al., 2010; FAO, 2013). Among the fermented milk product, yogurt is one of the most demanded products and considered as probiotic (Figueroa et al., 2011; Granato et al., 2010). It is considered as a highly valuable food product which is produced by the symbiotic association between the culture of Lactobacillus bulgaricus and Streptococcus thermophilus (Ramirez and Velez, 2013; Tripathi and Giri, 2014). This association prefer various health benefits and are coupled with improvement in nutritional value including fat contents reduction, hiding the textural consequences by adding proteins or exopolysaccharides (for LAB production or fermentation) or by adding probiotic microorganisms to afford definite nutrients for the microbiota of the gut and has a large number of consumers to pay for it (Vecchio et al., 2016). The functional and nutritional properties of the product depend upon the incorporated constituents and their effect on the water-holding capacity, texture, and other sensory properties. The consumer's interests for yogurt is high due to good taste, reduced cost contains all-natural ingredients in a single and easily available product. The herbal extract contains many phytoactive compounds with remedial properties but the

Corresponding author: Dr. Arvind

Assistant Professor, Department of Dairy Science and Food Technology, Institute of Agricultural Sciences, Banaras Hindu University, Varanasi221005, Uttar Pradesh, India

E-mail: arvind1@bhu.ac.in

Tel.: +91-9793583702 direct incorporation of these into the food product may disturb the flavor, taste, and texture of the product. Hence, to maintain the activity of these actives, the encapsulation approaches are used for entrapment (Mahdavi et al., 2014). The incorporation of active compounds into the foodstuffs as an entrapped particle is carried out for their stabilization, protection, and preservation (Zuidam and Shimoni, 2010). The competent transportation of bioactive compounds carried out using encapsulation approach by entrapment and preservation. Various applications of extracts with bioactive compounds with nutritional, functional, and remedial properties has established in the food sector. Accordingly, the present study was focused on the process optimization to develop the yogurt incorporated with encapsulated and non encapsulated Caesalpinia bonducella seed extract to show the effective use of encapsulation approach for hiding the taste and enhancing the texture and the bioavailability of the bioactive compounds and other nutritional properties of the herbal extracts.

\section{Materials and Methods}

Milk with two fat percentages (F1-1.5\% and F2-3.0\%) was collected from the nearby market of BHU, Varanasi, Uttar Pradesh. The extract of the Caesalpinia bonducella Flem. seeds were extracted in aqueous phase using ultrasonication and then encapsulated in a 1:2 ratio of sodium alginate and inulin, respectively by using a spray dryer. All the chemicals and reagents used in the study were analytical grade and procured from Sigma Chemicals Co. (St. Louise, M.O., USA); HiMedia Laboratories Pvt. Ltd., Bombay and Sisco Research Laboratories Pvt. Ltd., Bombay; Bangalore Genei, Bangalore, India. The optimized encapsulated and non encapsulated extract was subjected to incorporate into the yogurt @ 1.5 and 3.0\% fat (F1 and F2). The ten samples of yogurt including control (3 replicates

Copyright $@ 2020$ Ukaaz Publications. All rights reserved.

Email: ukaaz@yahoo.com; Website: www.ukaazpublications.com 
of each treatment) were prepared by taking the two ratios encapsulated, non encapsulated extract, and fat contents. Control with $1.5 \%$ fat contents (F1C), 1\% encapsulated extract (F1T1), 2\% encapsulated extract (F1T2), 1\% non encapsulated extract (F1T3), $2 \%$ non encapsulated extract (F1T4), Control with $3.0 \%$ fat contents (F2C), 1\% encapsulated extract (F2T5), 2\% encapsulated extract powder (F2T6), 1\% non encapsulated extract (F2T7), and 2\% non encapsulated extract (F2T8). The process of yogurt formation was carried out as shown in Figure 1.

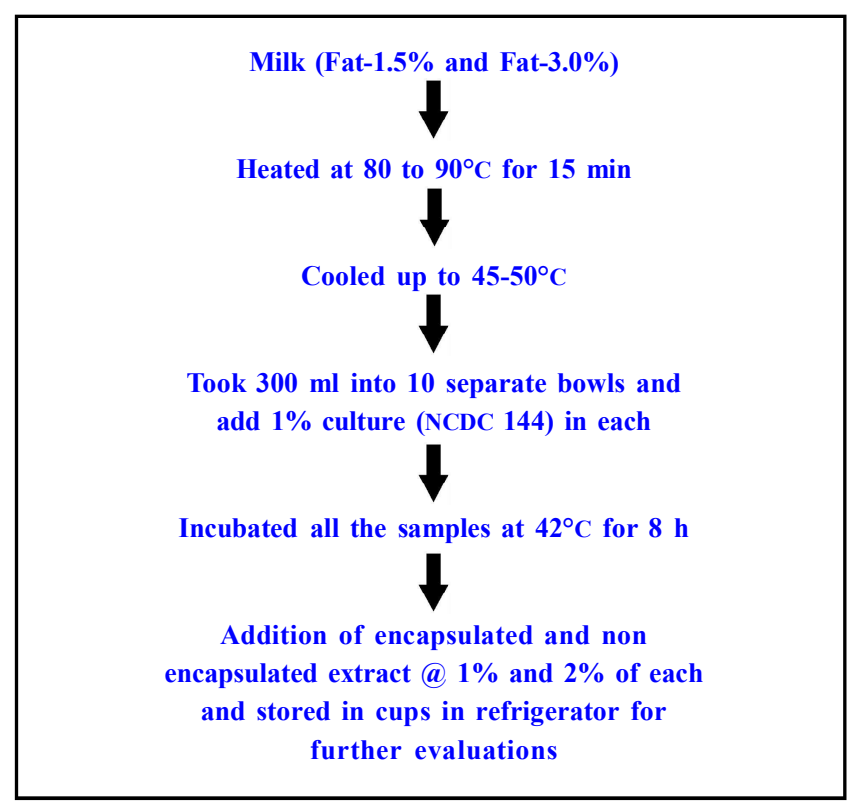

Figure 1: Flow diagram of yogurt formation with $1 \%$ and $2 \%$ encapsulated and non encapsulated C. bonducella Seed extract.

\subsection{Antioxidant evaluations}

The assessment of antiradical activity against DPPH was carried out by the subsequent method of Nishino et al. (2000) with few modifications in sample preparation and reaction amount. For analyzing the ABTS radical scavenging activity the method of Re et al. (1999) was followed with slight modifications. For analysis of the superoxide anion scavenging activity (SOSA) was done by the method of Liu et al. (1997) with little modifications. Total phenolic constituents of extracts were determined by methods involving Folin-Ciocalteu reagent and gallic acid standard according to the European Pharmacopoeia (Druckerei, 2002).

\subsection{Texture analysis}

The textural properties like hardness, Adhesiveness, Springiness, Cohesiveness, and Chewiness were analyzed by the texture profile analyzer (Model TA-XT stable microsystem Plus, UK). Back Extrusion probe (A/BE) with a $35 \mathrm{~mm}$ disc and extension bar using a $5 \mathrm{~kg}$ load cell was used. Tests were carried out in standard size back extrusion containers ( $50 \mathrm{~mm}$ diameter) with a sample size of $30 \mathrm{~mm}$ in height and width. The compressive force for the product (yogurt) by the probe was up to the twice distance of $20 \mathrm{~mm}$. The set conditions of texture analyzer for analyzing the textural characteristics are: the pre-test speed is $3 \mathrm{~mm} / \mathrm{sec}$; test and post-test speeds were $0.5 \mathrm{~mm} / \mathrm{sec}$, trigger force $5.0 \mathrm{~g}$ and time $5.0 \mathrm{sec}$ for each evaluation, about $30 \mathrm{~g}$ of yogurt was used during texture analysis.

\subsection{Sensory evaluation}

The optimized yogurt was analyzed for different sensory parameters such as color and appearance, flavor, body and texture, mouthfeel, and overall acceptability. The analysis was done by serving experimental and control samples after $4 \mathrm{~h}$ of incubation to reduce the temperature of yogurt by a panel of 12 semi-trained adjudicators (aged 25-65 years) from the Department of Dairy Science and Food Technology, IAS, Banaras Hindu University, Varanasi. Sensory evaluation was conducted at a temperature of 25 to $30^{\circ} \mathrm{C}$ by using a nine-point hedonic rating (9-point hedonic rating scale lies between, $1=$ dislike extremely to $9=$ like extremely) as explained by Amerine et al. (1965).

\subsection{Statistical analysis}

The data obtained during the present investigation was suitably analyzed by employing statistical designs. The results of preliminary studies are expressed as an average \pm standard error. Statistical significance was tested by employing the one-way analysis of variance (ANOVA) and comparison between means was made by the least significant difference and those at alpha and $p$-value $>0.05$.

\section{Results}

\subsection{Antioxidant and phenolic contents}

The antioxidant and phenolic contents of the yogurt samples incorporated with encapsulated and non encapsulated seed extract @ $1 \%$ and 2\% each are shown in Table 1. The yogurt sample (@3\% fat) incorporated with $2 \%$ extract had higher antioxidant and phenolic contents as compared with the others. The antioxidant activity and total phenolic contents value of both yogurts $(1.5 \%$ as well as $3.0 \%$ fat contents) with extract were higher than the yogurt with the encapsulated extract (Figure 2).

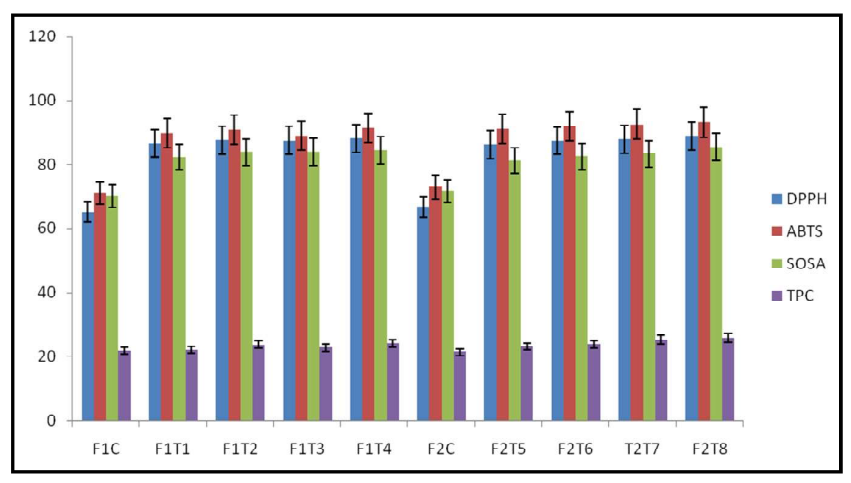

Figure 2: Antioxidants (DPPH inhibition, ABTS, and SOSA) and TPC evaluations of different yogurt samples incorporated with encapsulated and non encapsulated $C$. bonducella seed extracts.

\subsection{Texture profile analysis of yogurt}

The texture profile analysis of yogurt containing different percentages of encapsulated and non encapsulated extract of Caesalpinia bonducella seeds at two levels of fat percentage are shown in Table 2. Texture profile analysis of yogurt incorporated with different concentrations of encapsulated and non encapsulated extract was evaluated. The firmness of yogurt samples got lowered by enhancing the concentrations of encapsulated extract and fat levels. Conversely, the firmness of the $3 \%$ fat yogurt containing 
1 and $2 \%$ encapsulated extract is significantly lower than the $1.5 \%$ yogurt with 1 and $2 \%$ encapsulated extract. The firmness of the yogurt with extract was higher as compared to the firmness of the yogurt with the encapsulated extract. This may occur due to the weakness of the protein network, ensuring the reduction in firmness. Other texture properties including cohesiveness, springiness, gumminess, and chewiness also altered after adding the encapsulated and free extract.

\subsection{Sensorial evaluations of yogurt}

The sensorial evaluations of yogurt incorporated with various percentages of encapsulated and non encapsulated seed extract are presented in Table 3 . The yogurt incorporated with 1 and $2 \%$ of encapsulated and non encapsulated extract gave a statistically significant effect on sensory parameters including color and appearance, flavor, body, and texture, mouthfeel, and overall acceptability. The color and appearance score of control of both $1.5 \%$ and $3.0 \%$ fat level showed the lowest value of $7.58 \pm 0.09$ and $7.69 \pm 0.40$, respectively, as compared with the other sample containing the free and encapsulated extract. While on the other hand, the scores increased by increasing the level of encapsulated and non encapsulated seed extract. The color and appearance scores of the yogurt (both fat levels) with encapsulated seed extract was higher than the yogurt containing extracts at both concentration levels. The flavor score of yogurt with 2\% extract @both fat levels was $5.83 \pm 0.79$ and $5.43 \pm 0.96$, significantly less as compared with other yogurt samples including control. The flavor score of yogurt with encapsulated extract was significantly higher than the extract containing yogurt. The yogurt with 3\% fat had a higher flavor score as compared to the yogurt samples containing $1.5 \%$ fat content. The body and texture of the control yogurt (1.5\% fat) samples were slightly lower as compared to the control $(3.0 \%$ fat $)$. The addition of the extract at various percentages was significantly decreased the body and texture score of the yogurt. The score for the body and texture of the yogurt sample with $2 \%$ encapsulated powder was higher than the yogurt containing $1 \%$ encapsulated extract in both fat percentages. Figure 3 represents that the mouthfeel of the yogurt contains free extract in both fat percentages were less than the encapsulated extract. The yogurt sample with $2 \%$ encapsulated powder ( $3.0 \%$ fat level) had higher scores for the mouthfeel. The overall acceptability of the yogurt with $2 \%$ encapsulated extract (3.0\% fat) was the best over other samples represented by table 2 . Due to hiding the bitterness of the seed extract by encapsulation enhances the sensorial properties of the yogurt with encapsulated extract. The treatments of the 3.0\% fat yogurt had the best results as compared with the $1.5 \%$ fat yogurt.

Table 1: Antioxidants (DPPH inhibition, ABTS, and SOSA) and TPC assessments of different yogurt samples incorporated with encapsulated and non encapsulated C. bonducella seed extracts

\begin{tabular}{|l|l|l|l|l|l|}
\hline Fat level & Sa mples & DPPH (\% inhibition) & ABTS (\% inhibition) & SOSA (\% inhibition) & TPC (mg/g GA) \\
\hline $1.5 \%$ Fat & F1C & $65.14 \pm 0.29$ & $71.04 \pm 0.26$ & $70.09 \pm 0.39$ & $21.18 \pm 0.37$ \\
& F1T1 & $86.57 \pm 0.09$ & $89.82 \pm 0.36$ & $82.32 \pm 0.27$ & $21.98 \pm 0.08$ \\
& F1T2 & $87.59 \pm 0.27$ & $90.79 \pm 0.09$ & $83.71 \pm 0.29$ & $23.30 \pm 0.09$ \\
& F1T3 & $87.45 \pm 0.26$ & $88.98 \pm 0.55$ & $83.92 \pm 0.21$ & $22.69 \pm 0.04$ \\
& F1T4 & $88.82 \pm 0.17$ & $91.44 \pm 0.16$ & $85.54 \pm 0.17$ & $24.08 \pm 0.05$ \\
$3.0 \%$ fat & F2C & $66.76 \pm 0.85$ & $72.93 \pm 0.42$ & $71.71 \pm 0.48$ & $21.32 \pm 0.25$ \\
& F2T5 & $86.17 \pm 0.74$ & $91.09 \pm 0.08$ & $81.13 \pm 0.26$ & $23.11 \pm 0.06$ \\
& F2T6 & $87.38 \pm 0.04$ & $91.98 \pm 0.54$ & $82.41 \pm 0.08$ & $23.84 \pm 0.04$ \\
& F2T7 & $87.77 \pm 0.15$ & $92.54 \pm 0.40$ & $83.17 \pm 0.04$ & $25.30 \pm 0.12$ \\
CD @ 5\% & F2T8 & $88.12 \pm 0.42$ & $93.23 \pm 0.41$ & $84.48 \pm 0.40$ & $25.77 \pm 0.08$ \\
CD @ 5\% & Chromotype & N/S & 0.35 & 0.28 & 0.15 \\
\end{tabular}

F1C-cotrol for $1.5 \%$ fat yogurt, F1T1-1\% encapsulated extract, F1T2-2\% encapsulated extract, F1T3-1\% non encapsulated extract, F1T4-2\% non encapsulated extract, F2C-control for 3.0\% fat yogurt, F2T5-1\% encapsulated extract, F2T6-2\% encapsulated extract, F2T7-1\% non encapsulated extract, F2T8-2\% non encapsulated extract (all the treatments were in triplicates). All data are expressed as means \pm SD (n=3) with CD @ 5\% of both treatments and chromotype at alpha value $>0.05$.

Table 2: Texture profile analysis of different yogurt samples containing encapsulated and free $C$. bonducella Seed extracts

\begin{tabular}{|l|c|c|c|l|c|c|}
\hline Treatments & Hardness & Adhesiveness & Springiness & Cohesiveness & Gumminess & Chewiness \\
\hline F1C & $95.14 \pm 0.17$ & $871.25 \pm 0.22$ & $1.02 \pm 0.005$ & $0.867 \pm 0.047$ & $85.29 \pm 0.30$ & $86.47 \pm 0.36$ \\
F1T1 & $27.15 \pm 0.013$ & $225.28 \pm 0.27$ & $0.83 \pm 0.004$ & $0.563 \pm 0.017$ & $15.74 \pm 0.041$ & $13.02 \pm 0.026$ \\
F1T2 & $9.27 \pm 0.040$ & $1073.4 \pm 0.11$ & $1.54 \pm 0.043$ & $0.294 \pm 0.0013$ & $2.77 \pm 0.031$ & $4.38 \pm 0.041$ \\
F1T3 & $139.71 \pm 0.29$ & $652.36 \pm 0.44$ & $1.009 \pm 0.0017$ & $0.914 \pm 0.0014$ & $127.7 \pm 0.042$ & $128.41 \pm 0.52$ \\
F1T4 & $230.05 \pm 0.052$ & $911.32 \pm 0.24$ & $0.97 \pm 0.0012$ & $0.791 \pm 0.0012$ & $182.091 \pm 0.089$ & $178.51 \pm 0.38$ \\
F2C & $61.43 \pm 0.094$ & $1896.25 \pm 0.22$ & $0.99 \pm 0.0013$ & $0.192 \pm 0.0082$ & $11.81 \pm 0.013$ & $11.80 \pm 0.048$ \\
F2T5 & $2.45 \pm 0.028$ & $138.45 \pm 0.34$ & $0.48 \pm 0.0022$ & $0.785 \pm 0.0022$ & $1.93 \pm 0.023$ & $0.937 \pm 0.004$ \\
F2T6 & $2.25 \pm 0.047$ & $146.99 \pm 0.54$ & $0.503 \pm 0.0021$ & $0.833 \pm 0.047$ & $1.77 \pm 0.0026$ & $0.893 \pm 0.0017$ \\
F2T7 & $147.47 \pm 0.13$ & $527.85 \pm 0.26$ & $0.74 \pm 0.0082$ & $0.724 \pm 0.0013$ & $106.56 \pm 0.417$ & $79.06 \pm 0.073$ \\
F2T8 & $159.34 \pm 0.12$ & $3556.57 \pm 0.26$ & $0.98 \pm 0.0045$ & $0.329 \pm 0.0029$ & $52.27 \pm 0.21$ & $50.87 \pm 0.49$ \\
CD @ 5\% (Chromotype) & 0.114 & 0.266 & 0.013 & 0.020 & 0.170 & 0.264 \\
CD @ 5\% (Treatments) & 0.180 & 0.595 & 0.021 & 0.032 & & 0.269 \\
\hline
\end{tabular}

F1C-cotrol for $1.5 \%$ fat yogurt, F1T1-1\% encapsulated extract, F1T2-2\% encapsulated extract, F1T3-1\% non encapsulated extract, F1T4-2\% non encapsulated extract, F2C-control for 3.0\% fat yogurt, F2T5-1\% encapsulated extract, F2T6-2\% encapsulated extract, F2T7-1\% non encapsulated extract, F2T8-2\% non encapsulated extract (all the treatments were in triplicates). All data are expressed as means \pm SD (n=3) with CD @ 5\% of both treatments and chromotype at $p$-value $>0.05$. 
Table 3: Sensorial evaluations of different yogurt samples containing encapsulated with sodium alginate and inulin (1:2 ratio) and non encapsulated $C$. bonducella Seed extracts

\begin{tabular}{|l|l|l|l|l|l|}
\hline Treatments & Color and appearance & Flavor & Body and texture & Mouth feel & Overall acceptability \\
\hline F1C & $7.58 \pm 0.09$ & $7.65 \pm 0.44$ & $6.49 \pm 0.46$ & $6.78 \pm 0.45$ & $6.83 \pm 0.41$ \\
F1T1 & $8.06 \pm 0.33$ & $7.92 \pm 0.34$ & $7.51 \pm 0.33$ & $7.18 \pm 0.42$ & $7.49 \pm 0.31$ \\
F1T2 & $8.13 \pm 0.29$ & $8.04 \pm 0.48$ & $7.87 \pm 0.64$ & $7.81 \pm 0.33$ & $7.92 \pm 0.27$ \\
F1T3 & $7.73 \pm 0.37$ & $6.46 \pm 0.56$ & $6.36 \pm 0.46$ & $6.09 \pm 0.41$ & $6.21 \pm 0.83$ \\
F1T4 & $7.96 \pm 0.40$ & $5.83 \pm 0.79$ & $5.67 \pm 0.62$ & $5.64 \pm 0.48$ & $5.29 \pm 0.43$ \\
F2C & $7.69 \pm 0.94$ & $7.53 \pm 0.40$ & $7.39 \pm 0.39$ & $7.39 \pm 0.34$ & $7.49 \pm 0.26$ \\
F2T5 & $8.08 \pm 0.32$ & $8.15 \pm 0.27$ & $7.99 \pm 0.45$ & $7.66 \pm 0.31$ & $7.78 \pm 0.31$ \\
F2T6 & $8.15 \pm 0.32$ & $7.91 \pm 0.49$ & $8.04 \pm 0.32$ & $7.98 \pm 0.29$ & $8.16 \pm 0.36$ \\
F2T7 & $7.77 \pm 0.28$ & $6.35 \pm 0.41$ & $6.32 \pm 0.44$ & $5.99 \pm 0.49$ & $6.14 \pm 0.55$ \\
F2T8 & $7.98 \pm 0.43$ & $5.43 \pm 0.96$ & $5.38 \pm 0.94$ & $5.42 \pm 0.65$ & $4.95 \pm 0.75$ \\
p-value & 0.638 & 0.743 & 0.913 & 0.832 & 0.949 \\
\hline
\end{tabular}

F1C-cotrol for $1.5 \%$ fat yogurt, F1T1-1\% encapsulated extract, F1T2-2\% encapsulated extract, F1T3-1\% non encapsulated extract, F1T4-2\% non encapsulated extract, F2C-control for 3.0\% fat yogurt, F2T5-1\% encapsulated extract, F2T6-2\% encapsulated extract, F2T7-1\% non encapsulated extract, F2T8-2\% non encapsulated extract (all the treatments were in triplicates). All data are expressed as means \pm SD (n=12) with $p$-value $>0.05$.

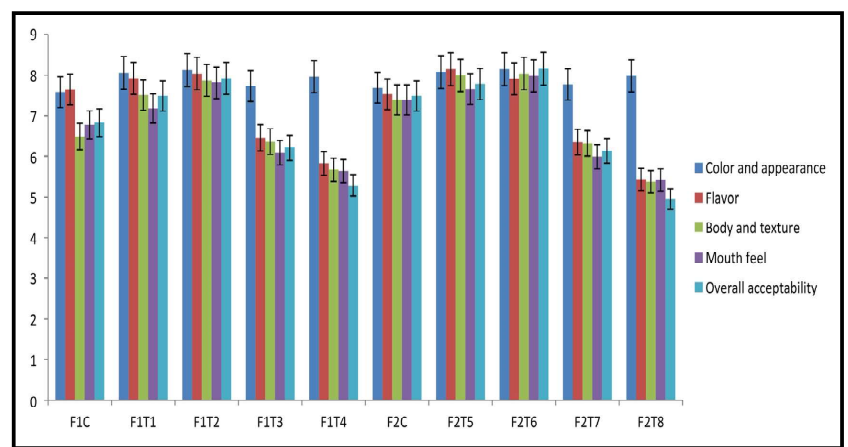

Figure 3: Sensorial analysis of different yogurt samples containing encapsulated and free $C$. bonducella seed extracts.

\section{Discussion}

The yogurt with extract had higher antioxidant and phenolic contents as compared to the yogurt with encapsulated extract may be due to the high temperature of spray dryer which is used for making a powder of encapsulated extract, generally, more than $60^{\circ} \mathrm{C}$ temperature reduces the activity of the bioactive compounds present in herbal extracts. The results were compared with a similar study in which the total polyphenol and flavonoid contents increased by increasing the levels of Aronia juice as compared with control (Nguyen and Hwang 2016). On the other hand, Servili et al. (2011) reported that the total phenolic contents of functional milk beverages fortified with olive oil extracts had $53.4-172.5 \mathrm{mg} / \mathrm{kg}$. Karaaslan et al. (2011) reported that the values of total phenolic contents (40-80 mg GAE/ Kg) of yogurts with grape and callus extracts. Another research evaluated that the products with jambolan powder had lower TPC, anthocyanins and DPPH inhibition activity as compared to the samples contains jambolan pulp (Bezerra et al., 2015). The antioxidant activity of the yogurt samples incorporated with free and encapsulated olive leaf extract was considerably higher than control yogurt (Tavakoli et al., 2018). The change in textural properties occurs due to the reduced potency of protein-protein, protein- lipid interactions. In a similar study, the textural properties of yogurt were increased by adding inulin and its hydrolysates at different concentrations (Yi et al., 2010). Alterations in the sensorial properties were compared with the similar studies evaluated that the yogurt containing 2\% Aronia juice had better taste than the 1 and 3\% Aronia juice while no significant difference was found in other sensorial properties among other samples (Nguyen and Hwang 2016). Tavakoli et al. (2018) reported that the addition of olive leaf extract in the form of nanoliposomes to the yogurt had the highest acceptability while the yogurt containing the free extract was lower. Hence, the above results indicated that the encapsulation strategy can minimize the unwanted feels (flavor, color, and mouthfeel) of the extract in the food products.

\section{Conclusion}

The antioxidant and total phenolic contents of the yogurt sample (@) both fat percentage) containing 2\% extract were highest but the sensory acceptance was very less as compared to others. The consumer acceptance score of the $3.0 \%$ fat yogurt with a $2 \%$ encapsulated extract was best among all in terms of taste and texture. The yogurt samples with $3.0 \%$ fat were better as compared to the yogurt samples with $1.5 \%$ fat. Employment of encapsulated C. bonducella seed extract in yogurts enhanced their textural characteristics. Sensorial evaluation of yogurts with encapsulated extract had an enhanced body, appearance, and smoother texture than yogurts with non encapsulated extract. Although, the antioxidant activity and phenolic contents of yogurt containing non encapsulated extract was higher the sensorial evaluations of the $3 \%$ fat yogurt with $2 \%$ encapsulated extract was best among all. Consequently, the present study was dedicated to the incorporation of the encapsulated Caesalpinia bonducella seed extract with remedial properties to facade the bitter taste and enhance the immovability of the herbal extract in yogurt system.

\section{Acknowledgments}

The authors acknowledge the Department of Dairy Science and Food Technology, I.A.S., BHU, Varanasi, for economic and research support for providing all the necessary research conveniences.

\section{Conflict of interest}

The authors declare that there are no conflicts of interest in the course of conducting the research. All the authors had final decision regarding the manuscript and decision to submit the findings for publication. 


\section{References}

Abadía-García, L.; Cardador, A.; del Campo, S.T.; Arvízu, S.M.; Castaño-Tostado, E.; Regalado-González, C.; García-Almendarez, B. and Amaya-Llano, S.L. (2013). Influence of probiotic strains added to cottage cheese on generation of potentially antioxidant peptides, anti-listerial activity, and survival of probiotic microorganisms in simulated gastrointestinal conditions. International Dairy Journal, 1;33(2):191-197.

Amerine, M.A.; Pangborn, R. and Roessler, E.B. (1965). Principles of Sensory Evaluation of Food. New York: Academic Press.

Ares, G.; Besio, M.; Giménez, A.; and Deliza, R. (2010). Relationship between involvement and functional milk desserts intention to purchase. Influence on attitude towards packaging characteristics. Appetite. 1;55(2):298-304.

Bezerra, M.; Araujo, A.; Santos, K. and Correia, R. (2015). Caprine frozen yoghurt produced with fresh and spray dried jambolan fruit pulp (Eugenia jambolana Lam) and Bifidobacterium animalis subsp. lactis BI-07. LWT-Food Science and Technology, 1;62(2):1099-104.

Druckerei, C.H. (2002). European Pharmacopoeia, 4th edn. Beck, Nordlingen, page No. 187.

Figueroa, R.; Tamayo J.; Gonzalez, S.; Moreno, G. and Vargas, L. (2011). Actividad antioxidante de antocianinas presentes en cáscara de pitahaya (Hylocereus undatus). Revista Iberoamericana de Tecnología Postcosecha, 12(1):44-50.

Granato, D.; Branco, G.F.; Nazzaro, F.; Cruz, A.G. and Faria, J.A. (2010). Functional foods and nondairy probiotic food development: Trends, concepts, and products. Comprehensive reviews in food science and food safety, 9(3):292-302.

IFAD W. FAO (2013). The State of Food Insecurity in the World: The multiple dimensions of food security.

Karaaslan, M.; Ozden, M.; Vardin, H. and Turkoglu, H. (2011). Phenolic fortification of yogurt using grape and callus extracts. LWT-Food Science and Technology, 1;44(4):1065-1072.

Liu, F.; Ooi, V.E.C. and Chang, S.T. (1997). Free radical scavenging activity of mushroom polysaccharide extracts. Life Science, 60:763-771.
Mahdavi, S.A.; Jafari, S.M.; Ghorbani, M. and Assadpoor, E. (2014). SprayDrying microencapsulation of anthocyanins by natural biopolymers: A review. Drying Technology, 32(5):509-518.

Nguyen, L. and Hwang, E.S. (2016). Quality characteristics and antioxidant activity of yogurt supplemented with aronia (Aronia melanocarpa) juice. Preventive Nutrition and Food Science, 21(4):330.

Ramirez-Sucre, M.O. and Velez-Ruiz, J.F. (2013). Physicochemical, rheological and stability characterization of a caramel flavored yogurt. LWTFood Science and Technology, 1;51(1):233-241.

Re, R.; Pellegrini, N.; Proteggente, A.; Pannala, A.; Yang, M. and Rice-Evans, C. (1999) Antioxidant activity applying an improved ABTS radical cation decolorisation assay. Free Radical Biol. Med. 26:1231-1237.

Servili, M.; Rizzello, C.G.; Taticchi, A.; Esposto, S.; Urbani, S.; Mazzacane, F.; Di Maio, I.; Selvaggini, R.; Gobbetti, M. and Di Cagno, R. (2011). Functional milk beverage fortified with phenolic compounds extracted from olive vegetation water, and fermented with functional lactic acid bacteria. International Journal of Food Microbiology, 14;147(1):45-52.

Tavakoli, H.; Hosseini, O.; Jafari, S.M. and Katouzian, I. (2018). Evaluation of physicochemical and antioxidant properties of yogurt enriched by olive leaf phenolics within nanoliposomes. Journal of Agricultural and Food Chemistry, 66(35):9231-40.

Tripathi, M.K. and Giri, S.K. (2014). Probiotic functional foods: Survival of probiotics during processing and storage. Journal of Functional Foods, 1;9:225-241.

Vecchio, R.; Van Loo, E.J. and Annunziata, A. (2016). Consumers' willingness to pay for conventional, organic and functional yogurt: Evidence from experimental auctions. International Journal of Consumer Studies, 40(3):368-378.

Yi, H.; Zhang, L.; Hua, C.; Sun, K. and Zhang, L. (2010). Extraction and enzymatic hydrolysis of inulin from Jerusalem artichoke and their effects on textural and sensorial characteristics of yogurt. Food and Bioprocess Technology, 1;3(2):315-319.

Zuidam, N.J. and Shimoni, E. (2010). Overview of microencapsulates for use in food products or processes and methods to make them. In Encapsulation technologies for active food ingredients and food processing. pp:3-29. Springer, New York, NY. 